\title{
Estudo de caso de um serviço de reabilitação auditiva na Cidade de São Paulo
}

\author{
Case study of a hearing rehabilitation service in the City of São Paulo \\ Estudio de caso de un servicio de rehabilitación auditiva en la Ciudad de São Paulo
}

Recebido: 23/07/2021 | Revisado: 30/07/2021 | Aceito: 03/08/2021 | Publicado: 08/08/2021

\author{
Cláudia Aparecida Ragusa-Mouradian \\ https://orcid.org/0000-0002-5902-1960 \\ Pontifícia Universidade Católica de São Paulo, Brasil \\ E-mail: claudia_ragusa@uol.com.br \\ Teresa Maria Momensohn-Santos \\ https://orcid.org/0000-0003-4751-0721 \\ Pontifícia Universidade Católica de São Paulo, Brasil \\ E-mail: teresa@ieaa.edu.br
}

\begin{abstract}
Resumo
Apresentar estudo de caso de um serviço de reabilitação auditiva do Município de São Paulo. Reorganização do processo de reabilitação auditiva do paciente, proposta pela Área Técnica da Pessoa com Deficiência da Secretaria Municipal de Saúde, introduzindo o Acolhimento Multiprofissional Compartilhado, com vistas ao estabelecido na Linha de Cuidados e do Projeto Terapêutico Singular. Anteriormente, os pacientes da região agendados para diagnóstico, cumpriam o seguinte Protocolo: 1. Médico otorrinolaringologista - Entrada no Serviço; 2. Avaliação Audiológica; 3. Otorrinolaringologista - diagnóstico e conduta; 4. Fonoaudiólogo - pré-molde e pré-seleção do aparelho; 5. Fonoaudiólogo - Teste e definição do Aparelho e 6. Entrega/Dispensação do Aparelho, sendo que tal processo da entrada à concessão dos dispositivos auditivos de amplificação levava até 12 meses. A reorganização aconteceu em 4 Fases: 1. (abril/2015) - agendamento para realização das Etapas 1, 2 e 3 do Protocolo; 2. (outubro/2015) - inclusão da Etapa 4 (1 otorrinolaringologista e 2 fonoaudiólogos); 3. (janeiro/2017) inclusão segundo otorrinolaringologista e única forma de entrada no serviço; 4. (abril/2017) - inclusão do Serviço Social. Com esse modelo de funcionamento, foi constatada a redução no número de retornos, otimização das agendas, melhoria do acesso ao serviço e redução do tempo entre a primeira consulta e a concessão dos dispositivos auditivos de doze (12) para quatro (4) meses. O processo do paciente para a reabilitação auditiva dentro do Serviço foi todo reestruturado e descrito, constituindo-se como um modelo a ser replicado. A reorganização constituiu-se como uma experiência de sucesso.
\end{abstract}

Palavras chave: Fonoaudiologia; Audiologia; Audição; Aparelhos auditivos; Reabilitação auditiva; Centros de reabilitação; Gestão de serviços de saúde.

\begin{abstract}
To present a case study of a hearing rehabilitation service in the city of São Paulo. Reorganization of the patient's auditory rehabilitation process, proposed by the Technical Area of The Person of Disability of the Municipal Health Department, introducing the Shared Multiprofessional Reception, with a view to the one established in the Care Line and the Singular Therapeutic Project. Previously, patients in the region scheduled for diagnosis complied with the following Protocol: 1. ENT physician - Service Entry; 2. Audiological Evaluation; 3. Otorhinolaryngologist - diagnosis and conduct; 4. Audiologist - premold and pre-selection of the device; 5. Audiologist - Test and definition of the Device and 6. Delivery/Dispensing of the Device. This process of entry to the concession of hearing aids took up to 12 months. The reorganization took place in 4 Phases: 1. (April/2015) - scheduling for the realization of Steps 1, 2 and 3 of the Protocol; 2. (October/2015) - inclusion of Stage 4 (1 otorhinolaryngologist and 2 audiologists); 3. (January/2017) - inclusion according to otorhinolaryngologist and only way of entering the service; 4. (April/2017) - inclusion of Social Work. With this operating model, it was observed a reduction in the number of returns, optimization of schedules, improvement of access to the service and reduction of time between the first consultation and the granting of hearing devices from twelve (12) to four (4) months. The patient's process for auditory rehabilitation within the Service was all restructured and described, constituting a model to be replicated. The reorganization was a successful experience.
\end{abstract}

Keywords: Speech Therapy; Audiology; Hearing; Hearing aids; Auditory rehabilitation; Rehabilitation centers; Health service management.

\section{Resumen}

Presentar un estudio de caso de un servicio de rehabilitación auditiva en la ciudad de São Paulo. Reorganización del proceso de rehabilitación auditiva del paciente, propuesta por el Área Técnica de La Persona de Discapacidad del Departamento Municipal de Salud, introduciendo la Recepción Multiprofesional Compartida, con vistas a la establecida en la Línea asistencial y el Proyecto Terapéutico Singular. Previamente, los pacientes de la región programados para el diagnóstico cumplían con el siguiente Protocolo: 1. Médico otorrinolaringólogo - Ingreso al Servicio; 2. Evaluación Audiológica; 3. Otorrinolaringólogo - diagnóstico y conducta; 4. Audiólogo - pre-moldeo y preselección del dispositivo; 5 . Audiólogo - Prueba 
y definición del Dispositivo y 6. Entrega/Dispensación del Dispositivo. Este proceso de ingreso a la concesión de audífonos tomó hasta 12 meses La reorganización se llevó a cabo en 4 Fases: 1. (Abril/2015) - programación para la realización de los Pasos 1, 2 y 3 del Protocolo; 2. (octubre/2015) - inclusión de la etapa 4 (1 otorrinolaringólogo y 21 audiólogos); 3. (enero/2017) - inclusión según otorrinolaringólogo y única forma de entrar en el servicio; 4. (abril/2017) - inclusión de Trabajo Social. Con este modelo operativo, se observó una reducción en el número de retornos, optimización de horarios, mejora del acceso al servicio y reducción del tiempo entre la primera consulta y el otorgamiento de audífonos de doce (12) a cuatro (4) meses. El proceso del paciente para la rehabilitación auditiva dentro del Servicio fue reestructurado y descrito, constituyendo un modelo a replicar. La reorganización fue una experiencia exitosa.

Palabras clave: Logopedia; Audiología; Audiencia; Audífonos; Rehabilitación auditiva; Centros de rehabilitación; Administración de servicios de salud.

\section{Introdução}

As previsões com relação ao aumento da população e da expectativa de vida, são de que até 2050 teremos o dobro da população acima de 60 anos e o triplo da população acima de 80 anos. Considerando que a perda auditiva é a principal causa de deficiência em homens maiores de 60 anos e a segunda causa mais comum, em termos de anos vivendo com uma deficiência, entre as mulheres desse grupo, tornam-se necessárias políticas públicas para diminuir possíveis fatores de risco e de agravos, bem como desenvolver diretrizes para a (re)habilitação (ONU, 2015).

A deficiência auditiva tem desdobramentos funcionais, emocionais, sociais e econômicos, que poderiam ser evitados ou minimizados com ações de promoção e proteção da saúde, detecção a tempo e intervenção. Segundo a Organização Mundial de Saúde (WHO, 2018), 466 milhões de pessoas tem algum tipo de perda auditiva ( $>6.1 \%$ da população mundial), dessas 34 milhões são crianças (7\%) até 15 anos e 432 milhões, adultos (93\%). Além disso, cerca de um terço das pessoas com mais de 65 anos de idade são afetadas. As estimativas apontam para um crescimento 630 milhões de pessoas com essas alterações em 2030, chegando a 900 milhões $(9,6 \%)$ em 2050.

Os dados divulgados no Hearing Day 2021 pela OMS para a região das Américas, apontaram que hoje temos 217 milhões de pessoas vivendo com perdas auditivas, sendo que em 2050 teremos 322 milhões, 80\% dessas pessoas vivem em países de baixa e média renda e a maioria não tem acesso a qualquer tipo de intervenção. A expansão do acesso e do cuidado requer investimentos, pois a deficiência auditiva instalada onera os cofres públicos. As projeções mostram que num período de 10 anos o retorno seria de US\$32 para cada dólar investido (WHO, 2021).

O Global Burden of Disease (Cieza, et al., 2020) em um primeiro estudo com a estimativa sobre a prevalência e o impacto dos anos vivendo com uma deficiência ou incapacidade (YLDs - years of life lived with disability) e a necessidade global de serviços de reabilitação, apontou que uma em cada três pessoas necessitam de algum tipo de reabilitação durante a sua doença ou lesão. Foram selecionadas 25 condições para essa análise, tendo nas alterações musculoesqueléticas sua maior contribuição. As deficiências sensoriais como auditiva e visual ficaram em $2^{\circ}$ lugar. No caso da deficiência auditiva, o uso de Aparelho de Amplificação Individual (AASI) pode reduzir até 59\% da incapacidade. Os dados contrariam a visão comum da reabilitação centrada num serviço especializado necessário para poucas pessoas, ou seja, a reabilitação precisa ser aproximada das comunidades como parte integrante da atenção primária à saúde para alcançar mais pessoas necessitadas.

No Brasil, a Constituição de 1988 instituiu o Sistema Único de Saúde (SUS) que tem como princípio garantir ao cidadão brasileiro a saúde como um direito universal. Segundo este documento os entes federados devem planejar e assegurar atendimento às pessoas com deficiência, dentre as suas competências.

Segundo os dados do último CENSO realizado em 2010 pelo Instituto Brasileiro de Geografia e Estatística - IBGE apontou que 23,9\% da população tem algum tipo de deficiência (45,6 milhões de pessoas), sendo 2.759.004 pessoas na cidade de São Paulo. As questões levantadas indagavam sobre dificuldades de ouvir, enxergar, andar e/ou aprender, podendo constar mais de uma. No caso da deficiência auditiva, 4,59\% das pessoas referiram algum tipo de alteração e, na análise por faixa 
etária, nota-se um aumento da percepção das limitações funcionais no processo de envelhecimento em todas as deficiências, sendo esse aumento superior para deficiência física/ motora e auditiva. (IBGE, 2010)

A análise desses dados é imprescindível para aprimorar o diagnóstico e planejamento das ações da Rede de Cuidados à Pessoa com Deficiência, permitindo visualizar as deficiências referidas por territórios e atuar de forma descentralizada. Os serviços do SUS de reabilitação com modalidade auditiva têm um histórico desde os anos 90, quando foram publicadas as primeiras portarias sobre sua estruturação, com designação de verbas para concessão de dispositivos de amplificação sonora para os usuários com deficiência auditiva.

Em 2000 com a publicação da Portaria Ministerial nº 432 (Brasil, 2000), dá-se início no país a política de concessão de aparelhos auditivos via Serviços Ambulatoriais de Saúde Auditiva de Alta Complexidade cadastrados/credenciados junto ao Ministério da Saúde. Ao longo dos anos diversas atualizações e ajustes nessa política foram sendo realizadas, sendo desenhadas as formas de acesso e itinerário para os pacientes com perda auditiva nas diversas instituições credenciadas.

Diante disso, considera-se relevante descrever tal processo histórico, bem como ilustrar com o processo de amadurecimento de um serviço de reabilitação auditiva.

\section{Objetivo}

Apresentar o estudo de caso de um serviço de reabilitação auditiva do Município de São Paulo.

\section{Contexto Histórico}

\section{A. Brasil}

Até a década de 1990 o paciente com deficiência auditiva sem condições de aquisição de Aparelho de Amplificação Sonora Individual (AASI), poderia receber doações pelas Secretarias Estaduais e Municipais de Saúde ou Bem Estar Social. $\mathrm{Na}$ época não havia um itinerário definido, já que o paciente com diagnóstico de deficiência auditiva e prescrição de AASI tinha que buscar por entidades filantrópicas, fundos sociais de solidariedade, dentre outros, que reuniam os exames realizados e faziam a compra de grandes quantidades via licitação. Tal processo de doação poderia demorar mais de cinco anos. Com essa demora entre dar entrada no pedido e o recebimento dos dispositivos de amplificação, muitas vezes, poderia ocorrer em agravo do grau da perda gerando adaptações insatisfatórias.

No ano de 2000, foi publicada a Portaria Ministerial no 432 (Brasil, 2000), que regulamentava a concessão de aparelhos auditivos pelo Sistema Único de Saúde (SUS). Tal prestação de serviço seria realizada em Serviços Ambulatoriais de Saúde Auditiva de Alta Complexidade cadastrados junto ao Ministério da Saúde, respeitando critérios pré-estabelecidos e a partir da solicitação do gestor local.

Em uma análise, para o Ministério da Saúde, sobre a produção ambulatorial em saúde auditiva do $1^{\circ}$ semestre de 2004, e registrada no Sistema de Informações Ambulatoriais - SIA/SUS, as autoras mostraram que existia um total de 138 estabelecimentos de saúde credenciados pelas Secretarias Estaduais de Saúde, nas 22 Unidades Federais - UF. Observaram ainda, que havia maior concentração destes serviços nas capitais das regiões sudeste e sul do país. Ressaltaram a ocorrência de grandes discrepâncias entre os procedimentos realizados Diagnóstico, Seleção, Concessão de AASI, Acompanhamento e Terapia Fonoaudiológica, mostrando uma fragmentação do atendimento ao usuário. Do total dos registros apenas 16\% das unidades registravam todos os procedimentos, $25 \%$ um único tipo de procedimento e 59\% até três procedimentos. (Daher e Pisaneschi,2010)

Diante de tais circunstâncias em 2004, o Ministério da Saúde ciente da necessidade de organização do atendimento às pessoas nos diferentes níveis de atenção no SUS, instituiu a Política Nacional de Atenção à Saúde Auditiva (Portaria MS/GM $n^{\circ}$ 2073), que previa a criação de redes hierarquizadas, regionalizadas e integradas, contemplando os diferentes níveis de 
atenção à saúde auditiva. Para sua operacionalização, publicou normas complementares para sua implantação e organização (Portarias SAS/MS nº 587 e 68) e operacionalização dos procedimentos (Portaria SAS/MS nº 589), reordenando e aprimorando o atendimento ambulatorial e hospitalar nos Serviços Especializados, incluindo a cirurgia de Implante Coclear, realizada no SUS desde 1999 (Portaria MS/GM n 1278).

Em 2012, em decorrência do Plano Nacional dos Direitos da Pessoa com Deficiência - Plano Viver sem Limite (Decreto 7612, de 17/11/2011), o Ministério da Saúde publicou a Portaria MS/GM nº 793/12, revogando as demais Portarias e instituindo a Rede de Cuidados à Pessoa com Deficiência. Esta rede tinha como finalidade ampliar o acesso, qualificar o atendimento, articular e integrar os serviços de saúde (da atenção básica, especializada e hospitalar) de forma a garantir a integralidade do cuidado às pessoas com deficiência temporária ou permanente; progressiva, regressiva ou estável; intermitente ou contínua. Os serviços de saúde denominados especializados foram compostos pelos Centros Especializados de Reabilitação (CER), pelos Serviços de Reabilitação habilitados em uma única modalidade e pelas Oficinas Ortopédicas. No mesmo ano foi publicada a Portaria MS/GM no 835/12 que prevê incentivos financeiros de investimento, não mais por produção, mas por custeio de acordo com a complexidade de modalidade CER - Centro Especializado em Reabilitação - de II a IV (auditiva + intelectual, visual e física).

Em 2017 foram organizadas seis Portarias de Consolidação dos Atos Normativos do Ministério da Saúde, divididas em eixos temáticos. Essas Portarias resultaram da análise de 17 mil portarias vigentes, em que menos de 5\% continham normas ainda válidas. Tais normas foram consolidadas no que vem sendo denominado como "Código do SUS" e visa facilitar a compreensão, melhorar a gestão das políticas públicas e dar maior transparência às regras para gestores, órgãos de controle e cidadãos. Os temas de tais Portarias estão descritos a seguir:

Portaria de Consolidação n⿳0 01: Consolidação das normas sobre os direitos e deveres dos usuários da saúde, a organização e o funcionamento do Sistema Único de Saúde.

Portaria de Consolidação n⿳0 02: Consolidação das normas sobre as políticas nacionais de saúde do Sistema Único de Saúde.

Portaria de Consolidação nº 03: Consolidação das normas sobre as redes do Sistema Único de Saúde.

Portaria de Consolidação nº 04: Consolidação das normas sobre os sistemas e os subsistemas do Sistema Único de Saúde.

Portaria de Consolidação n⿳0 05: Consolidação das normas sobre as ações e os serviços de saúde do Sistema Único de Saúde.

Portaria de Consolidação no 06: Consolidação das normas sobre o financiamento e a transferência dos recursos federais para as ações e os serviços de saúde do Sistema Único de Saúde.

No que tange à Rede de Cuidados da Pessoa com Deficiência do SUS, a portaria $\mathrm{n}^{\circ} .3$ contempla questões relacionadas a atenção à saúde na Atenção Básica, Especializada, Hospitalar, Urgência e Emergência. Tem como parte integrante e especializada os Centros Especializados de Reabilitação (CER), os Serviços de Reabilitação de modalidade única e as Oficinas de Reabilitação. Estes serviços deverão estar articulados entre si e com os demais componentes da Rede de Atenção à Saúde.

Vale ressaltar que os estabelecimentos de saúde habilitados em apenas um Serviço de Reabilitação (modalidade única) até a data anterior à publicação das Portarias GM/MS n 793 de 24 de abril de 2012 (PRT de Consolidação n 3/GM/MS, de 28 de setembro de 2017, Anexo VI) e GM/MS nº 835 de 25 de abril de 2012 (Consolidação nº 6/GM/MS, de 28 de setembro de 2017, TÍTULO VIII, Capítulo IV), seguirão as exigências técnicas estabelecidas quando da data de sua habilitação. Tais serviços poderão requerer a qualificação para CER, desde previsto no Plano de Ação Regional e de que estejam de acordo com as exigências da Portaria de Consolidação nº 3. (Ministério da Saúde, 2020).

\section{B. Município de São Paulo}

No Município de São Paulo as discussões sobre a organização da Saúde Auditiva são antigas, tendo sido alavancadas em 1998 com a promulgação da Lei Municipal no 12.556, que criou o "Programa de Saúde Auditiva para crianças" por meio 
do decreto $n^{\circ} 42.214$ de 2002. Entre outras coisas esse decreto apontou para a necessidade de organização de serviços de referência e contrarreferência, sistematização de informações e desenvolvimento de projetos entre Saúde e Educação e, também da constituição de um Grupo Técnico com profissionais de diferentes secretarias Municipais e Estaduais, entidades de classe e Universidades. (Manzoni e Almeida, 2010).

Em São Paulo, a Secretaria Municipal de Saúde (SMS) iniciou, desde 2005, a implantação dos Núcleos Integrados de Reabilitação (NIR) e dos Núcleos Integrados de Saúde Auditiva (NISA) a fim de garantir o acesso do munícipe a reabilitação, próximo de sua moradia, de forma articulada com a atenção básica e com serviços de maior complexidade, com vistas na constituição da rede de atenção. No caso dos NISA respeitavam a seguinte classificação: NISA I (básico) - avaliação audiológica e reabilitação auditiva, NISA II (média complexidade) - diagnóstico audiológico, concessão de AASI para pacientes >3 anos sem afecções associadas e reabilitação auditiva e NISA III (alta complexidade) - diagnóstico audiológico incluindo pacientes $<3$ anos e com afecções associadas, concessão de AASI e reabilitação auditiva.

$\mathrm{Na}$ medida em que os Serviços foram sendo credenciados no Município seja sob gestão Estadual: Irmandade da Santa Casa de Misericórdia de São Paulo, Hospital das Clínicas (HC) e Hospital São Paulo (HSP), ou Municipal: Centro Especializado Médico Aquino (CEMA), Divisão de Educação e Reabilitação dos Distúrbios da Comunicação (DERDIC), Centro Audição na Criança (CeAC), Santa Casa de Misericórdia de Santo Amaro ou Serviços próprios: Núcleo Integrado de Saúde Auditiva (NISA) II Penha e Pirituba foi sendo constituída e ampliada a Rede de Saúde Auditiva. A partir das diretrizes do Ministério da Saúde, profissionais do município junto com a Área Técnica de Saúde da Pessoa com Deficiência (Grupo de Trabalho) realizaram discussões e levantamento das necessidades das diferentes regiões da cidade, especialmente aquelas mais periféricas.

Os primeiros Serviços próprios do Município, NISA II Penha e Pirituba, iniciaram suas atividades em setembro de 2006, credenciados como Média Complexidade. Dentre as tarefas realizadas pelo Grupo de Trabalho de Saúde Auditiva, com profissionais da prefeitura de São Paulo, encontram-se os projetos para aquisição de equipamentos, implementação de Recursos Humanos via concurso público, organização de Cursos de Atualização sobre AASI e reabilitação auditiva, e credenciamento das Empresas para fornecimento de AASI com dispensa de licitação.

Esses núcleos foram consolidados pela Lei Municipal no 14.671 (14/01/2008), que criou o Programa Municipal de Reabilitação da Pessoa com Deficiência Física e Auditiva, regulamentada pelo Decreto 49.671 (25/06/2008).

Até 2012 as diretrizes ministeriais voltadas a atenção em reabilitação à Pessoa com Deficiência estavam pautadas em Portarias que davam suporte à organização de Redes de Reabilitação direcionadas a um determinado tipo de deficiência, seja ela física (MS/GM no 818/2001 e MS/SAS n¹85/2002), auditiva (MS/GM 2.073/2004, MS/GM 587/2004 e MS/SAS 589/2004, visual (MS/GM 3.128/2008) ou intelectual (MS/SAS1635/2002). A partir dos normativos destas portarias foram habilitados na cidade de São Paulo 11 serviços de reabilitação física (três próprios, dois conveniados com o município e seis sob gestão estadual); oito serviços de saúde auditiva (dois próprios, três conveniados com o município e três sob gestão do estado); um de reabilitação visual (gestão estadual). Na deficiência intelectual a Portaria propôs um novo procedimento para esta atenção, sem requerer a habilitação de serviços. (Secretaria Municipal da Saúde, 2016).

A cidade de São Paulo pertence a uma das Redes de Atenção à Saúde - RASS-6 do Estado de São Paulo, como parte das atividades propostas pela Portaria 793/2012 para Rede de Cuidados à Pessoa com Deficiência. Em 2013, foi constituído um Grupo Condutor com o objetivo de elaborar o diagnóstico, construir um plano de ação e monitorar as ações desenvolvidas para a organização da Rede. Este Grupo foi composto por representantes da Secretaria Municipal de Saúde: Área Técnica Saúde da Pessoa com Deficiência, Gerência de Controle e Regulação, Coordenação de Epidemiologia e Informação (CEINFO), Autarquia Hospitalar Municipal, Atenção Básica, Programa de Atendimento Domiciliar, Coordenadorias Regionais de Saúde (CRS) e Secretaria de Estado da Saúde/DRS-1. O Grupo contou, ainda, com a participação da Secretaria Municipal 
da Pessoa com Deficiência e Mobilidade Reduzida, do Conselho Municipal de Saúde e com representantes do Conselho Municipal da Pessoa com Deficiência.(PMSP, 2013)

Em 2016, a Área Técnica da Pessoa com Deficiência do Município de São Paulo com o objetivo de subsidiar e contribuir com a organização dos serviços e consolidação da rede, revisou e ampliou as diretrizes técnicas para o cuidado constantes no documento anterior "DIRETRIZES TÉCNICAS PARA GESTORES E PROFISSIONAIS NA ÁREA DA SAÚDE DA PESSOA COM DEFICIÊNCIA NO MUNICÍPIO DE SÃO PAULO", elaborado em 2011. Tal documento incorporou as diretrizes nacionais apresentadas no Plano Nacional Viver Sem Limites, outras diretrizes elaboradas pela Secretaria Municipal de Saúde que constavam do Plano de Ação da Rede de Cuidados à Pessoa com Deficiência, além das sugestões das diversas Coordenadorias Regionais de Saúde (CRS), Supervisões Técnicas (STS) e dos serviços especializados.(PMSP, 2016)

No Quadro1 é possível visualizar o número, a distribuição dos serviços de reabilitação e suas respectivas modalidades na cidade de São Paulo, bem como tipo de gestão e habilitação.

Importante ressaltar que a cidade conta com 19 serviços de reabilitação com modalidade auditiva e três serviços credenciados para Implante Coclear: Hospital das Clínicas, Hospital São Paulo e Irmandade Santa Casa de Misericórdia de São Paulo.

Quadro 1. Distribuição e caracterização dos serviços de reabilitação na cidade de São Paulo

\begin{tabular}{|c|c|c|c|c|c|c|c|c|c|c|}
\hline CNS & STS & SERVICO & twobe as & phica & arrucruar & Alopir tedion & AUORINA & vasual & abm arual & mantsoo \\
\hline suetsti & móaca & can timiant & II & $x$ & $x$ & $\times 2$ & & & 3000 & 30 \\
\hline suetsin & Nonve & Con antoun acvi & 1 & $x$ & & & & & steono & nes \\
\hline suessit & newa & an wseus & iii & $x$ & & & $x$ & $\bar{x}$ & stacho & $\sin$ \\
\hline suossin & wronsana & an suronimas & III & $x$ & $x$ & $x 1$ & $x$ & & $300 \mathrm{M}$ & sin \\
\hline sucesn & vea muoesti. & an ven muobs & H & $x$ & $x$ & $x 1$ & & & ndono & 39 \\
\hline neesin & newesta & dan nivio gined & iv & $x$ & $x$ & & $x$ & $x$ & nebeeo & $\sin$ \\
\hline suersit & ver mencusa & as vin seater & II & $x$ & & & $x$ & & molowo & $\sin$ \\
\hline subesn & vea mentusu & aAC/ benoic & II & & $x$ & & $x$ & & runcachos & 30 \\
\hline sucess & viea menturas & Moshras s" & 1 & & & & $x$ & & morkats: & $\sin$ \\
\hline sus & sros & ansea & $N$ & $x$ & $x$ & $x+1$ & $x$ & $x$ & ans & $\sin$ \\
\hline sis & sea & 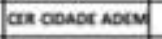 & $=$ & $x$ & $x$ & $x 1$ & & $x$ & sta cataken & $\sin$ \\
\hline sus & saca & an suvto aneser & in & $x$ & $x$ & $x_{2}$ & $x$ & & nowo & 30 \\
\hline sus & cavero tane & cin clverouned & $=$ & $x$ & $x$ & $x 2$ & $x$ & & móneso & $\sin$ \\
\hline su: & $\begin{array}{l}\text { Curtu Do } \\
\text { socosino }\end{array}$ & $\begin{array}{l}\text { Can naston } \\
\text { hibete }\end{array}$ & $N$ & $x$ & $\mathrm{x}$ & $x 1$ & $x$ & $x$ & ASF & No \\
\hline su & mativenas & Cin nastuatinos & $\pi$ & $x$ & $x$ & $x:$ & & & AS & Nio \\
\hline assin. & Weas & cresuan & 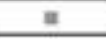 & $x$ & $x$ & $x+1$ & $x$ & & ASI & $\sin$ \\
\hline कest & sutavia & ax surkevih & 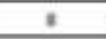 & $x$ & & & $x$ & & minno & $\sin$ \\
\hline astr & movetinos & trouvin & & & & & $x$ & & ton & $s \mathrm{se}$ \\
\hline canerwo & st & aras $x$ & 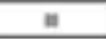 & $x$ & $x$ & $\times 2$ & $x$ & & wases & san \\
\hline astao & st & savta cask & & & & & $x$ & & savta Casa & $\sin$ \\
\hline woent & 10/penselvou & $\cos 10$ & 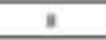 & $x$ & $x$ & $x_{2}$ & & & nobmo & $\sin$ \\
\hline soen & po/mensilvou & ara mania ciduf & 1 & $x$ & & & & & nowso & wo \\
\hline Nontr & naus & can naus & 1 & $x$ & & & & & nowno & wo \\
\hline Monte & $\begin{array}{l}\text { vi mukiant } \\
\text { cuevexnet }\end{array}$ & ora carenome & m & $x$ & $x$ & $x:$ & $x$ & & 3004 & sin \\
\hline vart & $\begin{array}{l}\text { Savinavi } \\
\text { Incend }\end{array}$ & an nociminn & 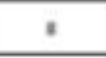 & $x$ & $x$ & $x:$ & & & noses & sis \\
\hline Nontr & $\begin{array}{l}\text { Ievinewar } \\
\text { incard }\end{array}$ & as incens & $\pi$ & $\mathrm{x}$ & $\mathrm{x}$ & $x 1$ & & & uses & wo \\
\hline vonte & nairves & an namus & 1 & & & & $x$ & & nobmo & sin \\
\hline Leste & maOUKA & cran NS Amantoed & $\pi$ & $x$ & $x$ & $x_{2}$ & & & móno & N10 \\
\hline Leith & coverusts & are so sotats & 8 & $x$ & $x$ & & & & sta. Manctuns & N10 \\
\hline Leste & cchentuses & cren cunisvases & $\pi$ & & & & $x$ & $x$ & Sth Mascauks & sim \\
\hline Leste & Sto veartivs & OS 10 vant & $\pi$ & $\mathrm{x}$ & $x$ & $x 1$ & & & HUNOACHOAR & No \\
\hline vente & $\begin{array}{l}\text { ockor } \\
\text { fasoevites }\end{array}$ & Cre tiansevits & 1 & $x$ & & & & & STA MAKOSOEA & wo \\
\hline Leste & Slo micun & ctan sho mecuth & $N$ & $x$ & $x$ & $\times 2$ & $x$ & $x$ & IFA Mascaush & $\sin$ \\
\hline Cente & 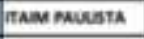 & Drom cunseco nd & $\pi$ & $x$ & $x$ & & & & STA Makctuna & No \\
\hline Levite & ravelaw & $\begin{array}{l}\text { ar caventivo } \\
\text { matravarzo }\end{array}$ & 1 & $x$ & & & & & mono & weo \\
\hline Leste & maga nausta & an 10 cesenos & 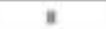 & $x$ & $x$ & $x_{1}$ & & & IFA Makcauks & No \\
\hline
\end{tabular}


Legenda: ASF - Associação Saúde da Família; CeAC - Centro de Audição na Criança; CEMA - Centro Especializado Médico Aquino; CER - Centro Especializado de Reabilitação; DERDIC - Divisão de Educação e Reabilitação dos Distúrbios da Comunicação; IABAS - Instituto de Atenção Básica e Avançada à Saúde; FORL - Fundação de Otorrinolaringologia; Fundação SP - Fundação São Paulo; Hospital SP - Hospital São Paulo; SECONCI-SP - Serviço Social da Construção - São Paulo; SPDM - Associação Paulista para o Desenvolvimento da Medicina; Sta. Catarina - Rede Santa Catarina Saúde; Sta. Marcelina - Atenção Primária à Saúde Santa Marcelina

\section{Proposta de Fluxo da Secretaria Municipal da Saúde}

No Município de São Paulo o acesso do usuário se dá por vagas disponibilizadas pelas unidades de referência e gerenciadas pela Central de Regulação das Coordenadorias Regionais de Saúde (CRS), sendo que algumas unidades disponibilizam tais vagas via reserva técnica do serviço. Esta reserva é determinada pela necessidade local, pela capacidade de atendimento do serviço e é disponibilizada no Sistema Integrado de Gestão de Assistência à Saúde de São Paulo (SIGA) de acordo com o protocolo pré-estabelecido. As Unidades de Saúde da rede de atenção as solicitam, mediante pedido do médico ou do Fonoaudiólogo. O agendamento é feito no SIGA, na Especialidade “Especialista em Reabilitação Auditiva”, no procedimento “Avaliação Multiprofissional em Reabilitação" (código: 0301079005). A regulação da CRS tem acesso exclusivo as vagas, gerencia a "Fila de Espera" e agenda por ordem de entrada no Sistema, considerando os critérios de prioridade estabelecidos pelo Grupo Técnico de Saúde Auditiva do Município. São consideradas prioridades:

- Crianças até 14 anos;

- Perdas súbitas como sequela de quadro infeccioso, como meningite, que possa levar a um processo degenerativo da fala;

- Seguido de casos de acordo com a sensibilidade do captador: assistente social, fonoaudiólogo, dentre outros;

- Adultos e/ou maiores de 14 anos em idade laborativa/estudos com perdas progressivas (moderadas - profundas), que estejam comprometendo a produtividade/qualidade de vida;

- Idosos com perdas adquiridas/progressivas com dificuldade de comunicação importante que cause isolamento / depressão.

A entrada nos serviços sempre se deu pela consulta do médico otorrinolaringologista, porém com a publicação da Portaria MS/GM n 793/12, instituindo a Rede de Cuidados à Pessoa com Deficiência, com os Instrutivos de Reabilitação, propôs a reorganização do acesso. Diante disso a Área Técnica da Pessoa com Deficiência da SMS orientou as Equipes, para que se organizassem para o acolhimento multiprofissional, a fim de reduzir os retornos ao máximo, com vistas ao estabelecimento da Linha de Cuidados e do Projeto Terapêutico Singular (PTS). Essa forma de entrada, prevê o agendamento via regulação de vagas do SIGA-Saúde (Sistema Integrado de Gestão de Assistência à Saúde de São Paulo), se constituindo ao longo do tempo como porta de entrada principal dos serviços.

A disponibilização das vagas segue os parâmetros sugeridos pela SMS de 40 casos novos/ mês, baseada na existência da equipe completa, conforme recursos humanos (RH) previsto pelo Ministério da Saúde (MS).

\section{Fluxo na Coordenadoria Regional de Saúde Norte (CRSN)}

A Coordenadoria de Saúde Norte (CRSN), conta com 2 (dois) serviços de referência para a concessão de AASI, são eles NISA Pirituba (2006) e CER III Carandiru (2015).

O acesso ao sistema de agendamento (regulação de vagas) se dá por meio da Unidade de Saúde da Rede de atenção, preferencialmente a Unidade Básica de Saúde (UBS) de origem do paciente, que solicita a vaga via Reserva Técnica (RT) do Serviço de referência, ou seja do Ambulatório de Especialidades de Pirituba/ NISA II Pirituba ou CER III Carandiru no SIGASaúde, cuja regulação de vagas é feita pela Coordenadoria de Saúde Norte (CRSN), mediante solicitação do médico ou 
Fonoaudiólogo. O agendamento é feito na Especialidade "Especialista em Reabilitação Auditiva", procedimento "Avaliação Multiprofissional em Reabilitação" (código: 0301079005).

A disponibilidade de vagas segue os parâmetros mínimos recomendados pela Secretaria Municipal de Saúde (SMS), considerando também o recurso financeiro para aquisição dos dispositivos de amplificação sonora.

Todo final de ano a Área Técnica da Pessoa com Deficiência da SMS faz uma proposta de empenho, considerando a série histórica da cidade, em termos de uso da verba destinada ao fornecimento de órteses, próteses e dispositivos de amplificação sonora. Essa séria histórica é baseada no uso da verba nos últimos anos e nas ocorrências que possam influenciar tal uso, como por exemplo o credenciamento de novas unidades ou quedas no rendimento de outras.

Essa verba é dividida por região, considerando o porte do serviço e sua capacidade de fornecimento dos dispositivos e uso dos recursos, baseado no tempo de credenciamento, experiência da equipe, recursos humanos (RH) e físicos disponíveis. A CRSN, recebeu no ano de 2018 a dotação equivalente a dois serviço e meio, sendo dividida da seguinte forma: o valor referente a um serviço e meio para o NISA II Pirituba e, de um serviço para o CER III Carandiru. Em números significa a concessão de cerca de 98 dispositivos para 49 pacientes por mês para o NISA II Pirituba e, respectivamente, 66 dispositivos para 33 pacientes por mês para o CER III Carandiru. Tais valores são previstos calculando-se a verba de acordo com a orientação do Ministério da Saúde, classificando os AASI em três tipos de Tecnologia: Tipo A (aparelhos mais simples e de tecnologia básica) - 50\%, Tipo B (tecnologia intermediária) -35\% e Tipo C (tecnologia avançada) - 15\% dos casos. Portanto, o valor total é distribuído respeitando tais porcentagens e dividido pelos meses do ano, chegando-se à quantidade de dispositivos passiveis de serem concedidos e para quantos pacientes.

A CRSN tem acesso exclusivo as vagas, gerencia a "Fila de Espera" e agenda por ordem de entrada no SIGA-Saúde, considerando os critérios de prioridade estabelecidos pelo Grupo Técnico de Saúde Auditiva do Município,

Além do acesso mediante agendamento via regulação de vagas, sendo essa a forma preconizada pela área técnica e a mais utilizada, o paciente pode eventualmente entrar por outras vias, como:

- Paciente em acompanhamento interno: Paciente em geral entrou via regulação de vagas, porém não era caso de adaptação de AASI, segundo os critérios da Portaria e foi mantido em acompanhamento no Serviço, sendo reintroduzido no processo quando necessário.

- Solicitação da diretoria ou outras instâncias, por ouvidoria ou outras queixas: Pacientes que são introduzidos no processo de concessão mediante solicitação da diretoria do Ambulatório de Especialidades (AE), Supervisão Técnica de Saúde (STS), Coordenadoria de Saúde Norte (CRSN) e/ou Secretaria Municipal da Saúde (SMS), em atendimento a solicitações judiciais, ouvidorias e processos administrativos.

- Encaminhamento interno direto: Pacientes que passaram em consulta com ORL, avaliação audiológica ou estão em processo terapêutico no NISA, que são encaixados diretamente no processo de concessão em decorrência de sua necessidade clínica (prioridade), psicológica ou limitações.

- Encaminhamento de outro Serviço com modalidade auditiva em geral por solicitação do paciente ou da família, para: Acompanhamento da adaptação ou anual, reposição de AASI e adaptação de FM

Para ilustrar, na Tabela 1 será apresentado o número de vagas oferecidas primeira vez, reposição e total no NISA II Pirituba, durante o ano de 2018. 
Tabela 1 - número de vagas oferecidas por mês para casos de primeira vez, reposição e total no NISA II PIRITUBA

\begin{tabular}{lccc}
\hline $\begin{array}{c}\text { Mês/2018 } \\
\text { vagas }\end{array}$ & $\mathbf{1}^{\text {a }}$ vez & rep osição & TOTAL \\
\hline Janeiro & 0 & 0 & 0 \\
Fevereiro & 30 & 10 & 40 \\
Março & 40 & 15 & 55 \\
Ab ril & 59 & 15 & 74 \\
Maio & 40 & 15 & 55 \\
Junho & 40 & 20 & 60 \\
Julho & 10 & 0 & 10 \\
Agosto & 38 & 14 & 52 \\
Setem bro & 20 & 20 & 40 \\
Outub ro & 45 & 17 & 45 \\
Novembro & 30 & 16 & 47 \\
Dezemb ro & 29 & $\mathbf{1 4 2}$ & 45 \\
TOTAL & $\mathbf{3 8 1}$ & & $\mathbf{5 2 3}$ \\
\hline
\end{tabular}

Fonte: Autores.

\section{Estudo de Caso}

Como o Núcleo Integrado de Saúde Auditiva (NISA) II Pirituba reduziu o número de retornos do paciente e otimizou o fluxo interno, por meio da organização do Acolhimento Multiprofissional Compartilhado, proposto pela Secretaria Municipal da Saúde (SMS), com vistas ao estabelecido na Linha de Cuidados e do PTS.

\section{Solução Encontrada para Melhorar o Fluxo Interno no Núcleo Integrado de Saúde Auditiva - NISA II Pirituba}

Em 2011, o Núcleo Integrado de Saúde Auditiva (NISA) Pirituba junto com a Coordenadoria Regional de Saúde Norte (CRSN), organizou uma forma de agendamento via reserva técnica, na regulação de vagas SIGA. Nesta proposta os pacientes da região eram agendados para a consulta médica e submetidos a avaliação diagnóstica dentro do Serviço. O Protocolo consistia em: 1. Consulta otorrinolaringológica - Entrada no NISA; 2. Avaliação Audiológica; 3. Consulta otorrinolaringológica para conclusão do diagnóstico e encaminhamento para seleção de aparelho; 4. Consulta Fonoaudiológica - Molde e seleção do aparelho; 5. Consulta Fonoaudiológica - Teste do Aparelho e 6. Consulta Fonoaudiológica Entrega/Dispensação do Aparelho, com isso o processo da entrada à concessão do AASI levava até 12 meses (Del Nero et al 2018).

Em 2015, diante da proposta da SMS de reorganização do acesso por meio de acolhimento multiprofissional compartilhado a fim de reduzir ao máximo o número de retornos, com vistas ao estabelecimento da Linha de Cuidados e constituição do PTS, o NISA II Pirituba, reorganizou o fluxo dos pacientes. Para a implantação desta proposta, passou por algumas fases para sua implantação:

FASE 1 -abril/2015 (Piloto): o agendamento dos pacientes foi realizado de forma que a consulta otorrinolaringológica acontecesse no mesmo dia que a avaliação audiológica (itens 1, 2 e 3 do protocolo). Os resultados mostraram ser possível reduzir em até 4 meses o tempo do paciente na Unidade.

FASE2-outubro/2015: com o sucesso do piloto, as agendas foram reorganizadas, incluindo-se a pré-seleção do aparelho e os pré-moldes (etapa 4 do Protocolo) no mesmo dia. Dessa forma o paciente passou a ser atendido pelos seguintes profissionais: um médico otorrinolaringologista (ORL) e dois fonoaudiólogos. 
FASE 3 - janeiro/2017: ampliação da forma de entrada incluindo o segundo médico ORL, contando com duas Equipes por semana e constituindo-se como única forma de entrada no serviço;

FASE4 - abril/2017: ampliação das Equipes com a inclusão da assistente social. Dessa forma o paciente passou a ser atendido pelos seguintes profissionais: um médico otorrinolaringologista (ORL) e dois fonoaudiólogos e um assistente social.

Essa reorganização da entrada, incluindo o maior número de avaliações no acolhimento multiprofissional compartilhado, permitiu que o paciente passasse pelas Etapas de 1 a 4 do protocolo inicial na primeira visita ao serviço, após cerca de 30 dias comparecesse para a Etapa 5 - Teste de AASI e, depois de aproximadamente 60 dias desta data, pela Etapa 6 - Entrega de AASI. Com esse modelo de funcionamento, foi constatada a redução no número de retornos, otimização das agendas, melhoria do acesso ao serviço e redução do tempo entre a primeira consulta e a concessão do aparelho auditivo de 12 para quatro meses. (Ragusa-Mouradian, 2018).

\section{Resultados e Discussão}

A reorganização do processo de reabilitação auditiva do paciente no NISAII Pirituba, que permitiu atender as solicitações da Área Técnica da Pessoa com Deficiência da SMS, tem várias etapas e pode ser visualizado na figura 1, sendo também descritas na sequência.

Figura 1. Fluxograma do processo do paciente no NISA II Pirituba

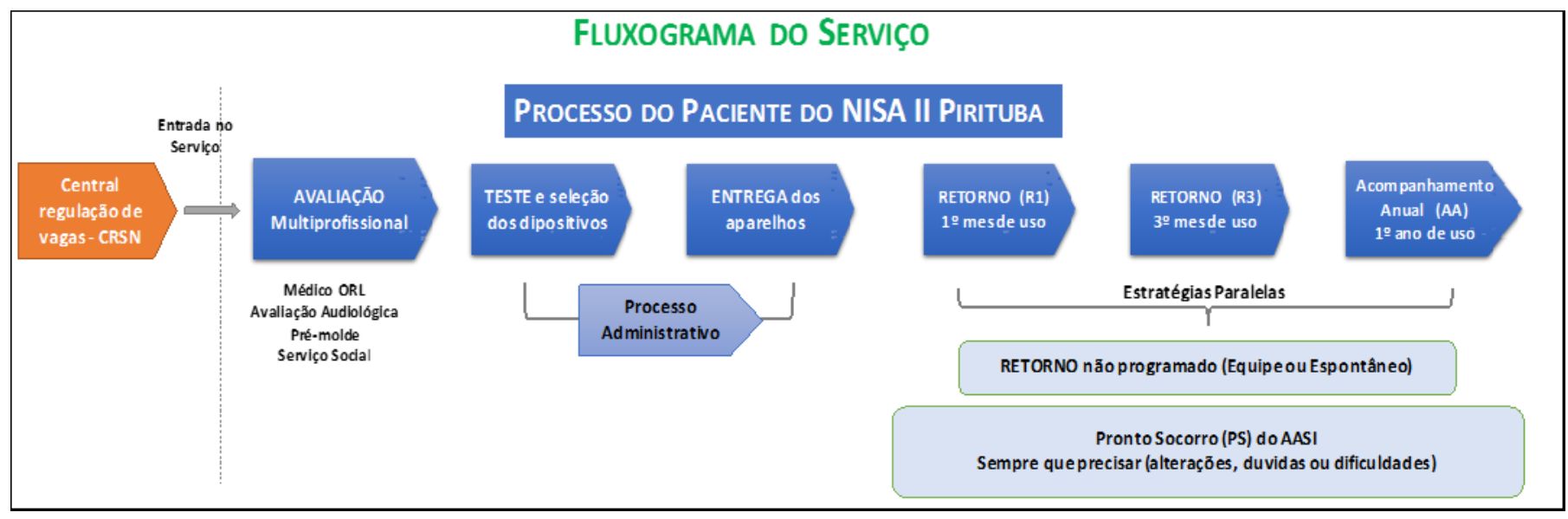

Fonte: Autores.

Etapa 1. Avaliação Multiprofissional do Paciente em Reabilitação Auditiva: Na data agendada o paciente comparece com a "Ficha de Encaminhamento" e passa por quatro consultas: 1. médico otorrinolaringologista (ORL), 2. Fonoaudiólogo 1 Avaliação Audiológica, 3. Fonoaudiólogo 2 - Molde (Assessoramento, pré-Seleção do AASI e pré-molde e agendamento do teste de AASI), e 4. Assistente Social.

Etapa 2. Teste e Seleção do AASI: Definição do modelo do AASI e continuidade do processo. Uma vez definido o AASI, o paciente é orientado a aguardar telefonema de agendamento para entrega dos AASI em até dois meses.

Etapa 3. Processo administrativo: parte burocrática de compra dos dispositivos, que inclui a autorização do Procedimento de Alta Complexidade (APAC) no sistema (SIGA); Emissão de Ordem de Fornecimento (OF); Emissão da nota fiscal (NF) e entrega dos aparelhos, kits e acessórios pela Empresa no serviço; Organização dos kits para entrega e transferência dos ajustes definidos na data do teste (armazenados no sistema NOAH) para os AASI(s) definitivos (programação), pela Equipe de fonoaudiólogos; Confecção dos Termos de Compromisso (individual) e agendamento das Entregas. 
Importante ressaltar que os dispositivos são adquiridos com dispensa de licitação, sendo realizado um edital de credenciamento das empresas pela Área Técnica da Pessoa com Deficiência da SMS para fornecimento dos mesmos, renovado anualmente durante cinco anos.

Etapa 4. Entrega dos AASI (s): pode ser individual, em dupla ou em grupo, para recebimento dos AASI e kits com os acessórios. As fonoaudiólogas fornecem orientações básicas de uso, manuseio, limpeza e a data do agendamento do Retorno de $1^{\circ}$ mês de uso - R1.

Etapa 5. Monitoramento do uso do AASI - acompanhamento e aconselhamento durante o processo de adaptação:

A. Consultas programadas -segundo o Protocolo do Serviço, durante o processo de adaptação o paciente deverá comparecer nas seguintes consultas:

- Retorno $1^{\circ}$ mês de uso - R1: orientação de uso dos acessórios de manutenção e limpeza, esclarecimento de dúvidas e reforço das orientações prévias;

○ Retorno $3^{\circ}$ mês de uso - R3: validação do processo, ajustes e avaliação do registro do Data Logging (DL), entrega da segunda cartela de baterias;

- Acompanhamento Anual - AA: Avaliação Audiológica, verificação, validação, ajustes e registro Data Logging (DL);

B. Consultas não programadas (extra protocolo) - por recomendação da Equipe ou procura espontânea do paciente:

- Retorno: agendado pela Equipe em caso de necessidade ou por procura espontânea, para realização de ajustes, dúvidas de uso, manuseio e limpeza, problemas de acoplamento, entre outros;

- Pronto Socorro (PS) do aparelho: acolhimento semanal de porta aberta resolução de dúvidas de uso, manuseio e limpeza, problemas de acoplamento (molde) ou com os AASI(s) a fim de evitar descontinuidade do uso e sobrecarga das agendas.

O acolhimento multiprofissional compartilhado permitiu uma melhor organização interna do serviço e das agendas, reduzindo o número de retornos do paciente, com a otimização dos procedimentos realizados em cada comparecimento na unidade, além de favorecer as discussões de caso e a atuação em equipe durante todo o processo. Com isso, o tempo entre a entrada no serviço e a concessão dos dispositivos, que era de dez a 12 meses, foi reduzida para cerca de 4 meses. Tal medida viabilizou a reabilitação auditiva de forma mais rápida e efetiva, endossando a Linha de Cuidados proposta pela SMS e a formalização do Projeto Terapêutico Singular (PTS). (Ragusa-Mouradian et al, 2018)

Na Figura 2 é possível visualizar o ganho em termos de tempo e a redução dos retornos entre a entrada no serviço e a entrega do AASI. 
Figura 2. Representação visual do processo de concessão do AASI antes e depois da implantação do Acolhimento multiprofissional compartilhado: é possível visualizar a Redução do tempo do tempo entre a Entrada no NISA e a entrega do AASI de 10 a 12 meses para até 4 meses.

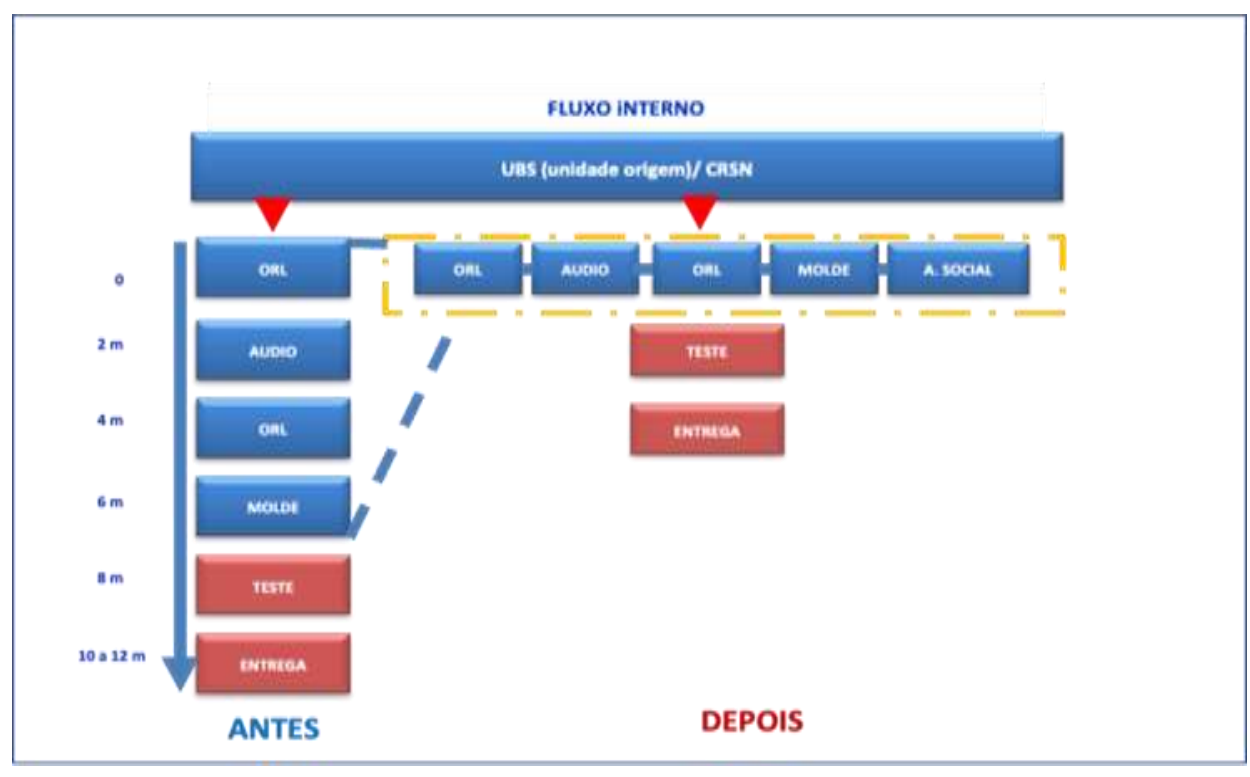

Fonte: Autores.

\section{Considerações Finais}

A reestruturação do processo de reabilitação auditiva no NISA II Pirituba, com vistas na proposta da Secretaria Municipal da Saúde (SMS) em termos de implantar o Acolhimento Multiprofissional Compartilhado, mostrou-se bemsucedida. A medida reduziu o número de retornos do paciente a unidade, sem comprometer o protocolo, otimizando as agendas, permitindo a melhoria do acesso e da organização do serviço, favorecendo ainda a atuação em equipe, já que a entrada do paciente é realizada e discutida em conjunto.

Além disso, o tempo entre a entrada no serviço ( $1^{\text {a }}$ consulta) e a concessão dos dispositivos (entrega) foi reduzido de 10 a 12 meses (processo anterior) para quatro meses, viabilizando a reabilitação mais rápida e efetiva, endossando a Linha de Cuidados proposta pela SMS e a formalização do PTS.

Visando a melhoria contínua do serviço, a ocorrência de reuniões mensais para alinhamento de condutas, discussão de casos, otimização do funcionamento, procedimentos e protocolos, foi implementada com todos os profissionais da a equipe do NISA. Paralelamente, a Coordenação do Serviço (Responsável Técnico) sempre participou ativamente das reuniões mensais de Reabilitação com as Equipes dos territórios das Supervisões Técnicas de Saúde (STS) Pirituba e Perus e das reuniões bimensais da Coordenadoria Regional de Saúde Norte (CRSN), num trabalho coletivo e integrado permitindo a aproximação dos diferentes atores envolvidos na organização e gestão, como também dos profissionais que atendem os pacientes, favorecendo a compreensão dos fluxos e protocolos de encaminhamento, discussão de casos, fortalecimento da Linha de Cuidados e construção do PTS, num processo de educação permanente e disseminação de melhores práticas.

\section{Conclusão}

A nova forma de agendamento constituiu-se como uma experiência de sucesso, já que houve diminuição na frequência do paciente no Serviço e do tempo entre a entrada e a dispensação dos dispositivos auditivos, favorecendo o aumento da oferta de vagas, além de estar em consonância com a proposta da SMS, constituindo-se como um modelo a ser replicado. 


\section{Referências}

Brasil. Constitução Federal de 1988. , (1988). Brasilia

Brasil. (2010). IBGE: Censo 2010. Retrieved March 31, 2021, from Instituto Brasileiro de Geografia e Estatística website: https://censo2010.ibge.gov.br/ Brasil. Decreto $N^{o} 7612$, (2011).

Brasil. Portaria No 793, De 24 De Abril De 2012. , Ministério Da Saúde. Gabinete Do Ministro § (2012).

Brasil. Portaria No 835, De 25 De Abril De 2012. , Ministério Da Saúde. Gabinete Do Ministro § (2012).

Brasil, M. Da S. Portaria No 2.073, De 28 De Setembro De 2004. , Diário Da República, $1^{\text {a }}$ Série - Nº 116 § (2004).

Cartilha Do Censo 2010 - Pessoas Com Deficiência (1 ${ }^{\mathrm{a}}$ Edição). (2012). Retrieved From Http://Www.Pessoacomdeficiencia.Gov.Br

Cieza, A., Causey, K., Kamenov, K., Hanson, S. W., Chatterji, S., \& Vos, T. (2020). Global Estimates Of The Need For Rehabilitation Based On The Global Burden Of Disease Study 2019: A Systematic Analysis For The Global Burden Of Disease Study 2019. The Lancet, 396(10267), $2006-2017$.

Https://Doi.Org/10.1016/S0140-6736(20)32340-0

Daher, C. V., \& Pisaneschi, E. (2010). A Politica Nacional De Atenção À Saúde Auditiva: A Atenção Especializada Às Pessoas Com Deficiência Auditiva No Sus. In S. (Organizadores) Bevilacqua, M. C.; Martinez, M. A. N.; Balen, S. A.; Pupo, A. C.; Reis, A. C. M. B.; Frota (Ed.), Saúde Auditiva No Brasil: Políticas, Serviços E Sistemas (Pp. 15-29). Retrieved From Http://Pesquisa.Bvsalud.Org/Bvsms/Resource/Pt/Mis-36294

Del Nero, A. L., Galdino, C.L.E., Ragusa-Mouradian, C.A., Silva, G.S., \& Bueno, V.S, (2018, Abril). Atendimento Multiprofissional Compartilhado: Otimozação Do Tempo Entre Entrada No Serviço E Concessao Do Aparelho Auditivo No Nisa Ii Pirituba. Anais Do $32^{\circ}$ Congresso De Secretários Municipais Da Saúde Do Estado De São Paulo, São Paulo, Sp, Brasil, 986-987.

Manzoni, C. R. C. T., \& Almeida, S. M. V. T. (2010). Organização Da Rede Se Atenção A Saúde Auditiva. In S. Bevilacqua, M. C.; Martinez, M. A. N.; Balen, S. A.; Pupo, A. C.; Reis, A. C. M. B.; Frota (Ed.), Saúde Auditiva No Brasil: Políticas, Serviços E Sistemas (Pp. 65-96). São José Dos Campos: Pulso Editorial.

Ministerio Da Saúde. (2020). Rede de Cuidados à Pessoa com Deficiência no Âmbito do Sus Instrutivos De Reabilitação Auditiva, Física 1 , Intelectual 2 E Visual (Centro Especializado Em Reabilitação-Cer E Oficinas Ortopédicas)., Nations, U. (2015). World Population Prospects: The 2015 Revision. United Nations Economic And Social Affairs, Xxxiii(2), 1-66. Retrieved From Http://Www.Americanbanker.Com/Issues/179_124/Which-City-Is-The-Next-BigFintech-Hub-New-York-Stakes-Its-Claim-1068345-1.Html\%5cnhttp://Www.Ncbi.Nlm.Nih.Gov/Pubmed/15003161\%5cnhttp:// Cid.Oxfordjournals. Org/Lookup/ Doi/10.1093/ Cid/Cir991\%5cnhttp://Www.Scielo

Ministerio Da Saúde. (2017). Portarias De Consolidação. Contratualizacaosus. (N.D.). Retrieved March 31, 2021,

Https://Www.Contratualizacaonosus.Com/Copia-Manuais-Do-Ministerio-Da-Saude

Prefeitura Do Municipio De São Paulo. (1998). Lei $N^{\circ} 12.556$, De 8 De Janeiro De 1998.

Prefeitura Do Municipio De São Paulo. (2002). Decreto $N^{o} 42.214$.

Prefeitura Do Municipio De São Paulo. (2013). Plano De Ação Da Rede De Cuidados Da Pessoa Com Deficiência.

Prefeitura Do Municipio De São Paulo. (2016). Diretrizes Para A Organização Das Ações De Reabilitação Na Rede De Cuidados À Pessoa Com Deficiência.

Ragusa-Mouradian, Claudia Aparecida;Galdino, Cibelle De Longhi Espassatempo; Ligia, Ricioli, Elena Silva; Silva, Gislaine Dos Santos; Del Nero, Ana Lucia; Coelho, Tatiana Gregório; Bueno, V. S. (2018). Avaliação Multiprofissional Compartilhada: Otimizando O Tempo No Processo De Reabilitação (Anais Do Congresso Sociedade Brasileira De Fonoaudiologia, Ed.). Http://Sbfa.Org.Br/Portal/Anais2018/Trabalhos_Select.Php?Id_Artigo=10673\&Tt= Sessão De Pôsteres

Ragusa-Mouradian, C. A. (2018). Avaliação Multiprofissional: Otimizando O Tempo E Os Retornos Desde A Entrada No Serviço Até A Concessão Do Aparelho Auditivo No Nisa Ii Pirituba. In E. E P. Em S. Secretaria Municipal Da Saúde, Coordenação De Gestão De Pessoas, Centro De Desenvolvimento (Ed.), Anais Dos Resumos Dos Trabalhos Apresentados No Congresso Municipal Da Rede De Atenção À Saúde Na Cidade De São Paulo: Desafios Da Organização Do Trabalho E Da Educação Na Saúde (Pp. 277-279). Https://Docs.Bvsalud.Org/Biblioref/2018/09/912500/Anais_Resumos_

Trabalhos_Congresso_Municipal_Ras.Pdf

World Health Organization. (2021). Highlighting Priorities For Ear And Hearing Care - World Report On Hearing. Retrieved March 31, 2021, From World Health Organization Website: Https://Www.Who.Int/Activities/Highlighting-Priorities-For-Ear-And-Hearing-Care

World Health Organization. (2018). Addressing The Rising Prevalence Of Hearing Loss.

Https://Apps.Who.Int/Iris/Bitstream/Handle/10665/260336/9789241550260-Eng.Pdf?Sequence=1\&Isallowed=Y

\section{Glossário}

CENSO: palavra vem do latim censos e significa "conjunto de dados estatísticos dos habitantes de uma cidade, província, estado, nação." É a principal fonte de dados sobre a situação de vida da população nos municípios e localidades.

Complexo Regulador do Município de São Paulo: é composto por estruturas denominadas Centrais de Regulação, que efetuam as ações do processo regulatório de acesso, ou seja, recebem, avaliam e autorizam e/ou agendam os atendimentos solicitados. As solicitações feitas às Centrais de Regulação do Complexo Regulador são avaliadas de acordo com diversos critérios técnicos, como a urgência clínica, grades de referências, níveis de complexidade, protocolos, ordem cronológica e agendadas à medida em que há disponibilidade de oferta. Quando há inconsistências, são devolvidas à unidade solicitante para correção e/ou complementação de informações.

Data Logging (DL): registro de dados dos aparelhos auditivos, que permitem verificar o número de horas de uso e ambientes frequentados. 
Research, Society and Development, v. 10, n. 10, e199101018633, 2021

(CC BY 4.0) | ISSN 2525-3409 | DOI: http://dx.doi.org/10.33448/rsd-v10i10.18633

Linha de cuidados: representa um continuum assistencial composto por ações de promoção, prevenção, tratamento e reabilitação". É o itinerário que o usuário faz por dentro de uma rede organizada de saúde.

Plano Terapêutico Singular (PTS):é um conjunto de propostas de condutas terapêuticas articuladas, para um sujeito individual ou coletivo, resultado da

discussão coletiva de uma equipe interdisciplinar, com apoio matricial, se necessário. Geralmente, é dedicado a situações mais complexas. Em verdade, é uma variação da discussão de "caso clínico (BRASIL, 2007).

Regulação de Vagas: é um processo essencial na garantia ao acesso universal e integral dos cidadãos aos cuidados de saúde, assegurado pela Constituição de 1988. A ação regulatória, quando aplicada de maneira adequada, otimiza os recursos disponíveis e favorece a devida entrada dos usuários ao Sistema Único de Saúde (SUS).

Reserva Técnica: são vagas que só podem utilizadas pela Central de Regulação, após analise quanto ao preenchimento dos requisitos e critérios préestabelecidos.

O SIA/SUS (Sistema de Informações Ambulatoriais): sistema de informações que registra a quantidade e tipo de procedimentos realizados pela unidade de saúde, tais dados são analisados com as demais informações, uma vez que não são suficientes para avaliar a qualidade do atendimento prestado.

SIGA-Saúde: é um portal/ ferramenta utilizada pelas unidades para o agendamento em tempo real das diversas ofertas em saúde das unidades executantes sob gestão municipal (próprios e contratados), e também como instrumento do processo regulatório para a disponibilização da oferta regionalizada, autorização dos procedimentos ambulatoriais de alto custo/complexidade e gerenciamento da demanda reprimida. 